\title{
Symmetry of boundary conditions of the Dirac equation for electrons in carbon nanotubes
}

\author{
Edward McCann and Vladimir I. Fal'ko \\ Department of Physics, Lancaster University, Lancaster, LA1 4YB, United Kingdom
}

\begin{abstract}
We consider the effective mass model of spinless electrons in single wall carbon nanotubes that is equivalent to the Dirac equation for massless fermions. Within this framework we derive all possible energy independent hard wall boundary conditions that are applicable to metallic tubes. The boundary conditions are classified in terms of their symmetry properties and we demonstrate that the use of different boundary conditions will result in varying degrees of valley degeneracy breaking of the single particle energy spectrum.
\end{abstract}

\section{INTRODUCTION}

Carbon nanotubes are the subject of intense research, motivated by the desire to use their unique physical and electronic properties in the development of nanoscale electrical devices[1,2]. The electronic properties of nanotubes follow from the band structure of graphene - a two-dimensional (2D) sheet of graphite - which is a semimetal, having a vanishing energy gap at the six corners, K-points, of the hexagonal first Brillouin zone. A singlewall nanotube may be thought of as a graphene sheet rolled up to form a nanometre-diameter cylinder. Periodicity around the circumference results in quantized transverse wavevectors leading to metallic or semiconducting behaviour depending on whether the K-point wavevector $\mathbf{K}$ is an allowed wavevector.

While the energy spectrum of an infinitely long tube will be continuous, a finite tube should possess discrete energy levels corresponding to standing waves typical of a confined quantum particle. Evidence of discrete levels was seen in transport measurements $[3,4]$ a few years ago, followed by the direct observation of sinusoidal standing wave patterns by scanning tunneling microscopy $[5,6]$. The measured wavelength of the standing waves $\lambda \sim$ $0.75 \mathrm{~nm}$, about three times larger than the lattice constant $a \approx 0.25 \mathrm{~nm}$, corresponded to wavevectors near the K-point K. More recently, Coulomb blockade measurements on carbon nanotube quantum dots $[7,8,9]$ have found evidence for fourfold periodicity of the spectra that is in agreement with expectations based on spin and $\mathrm{K}$ point degeneracy, although the experiments appeared to show varying degrees of degeneracy breaking. A number of authors $[10,11,12,13,14]$ have modelled finite-length nanotubes in order to describe the atomic scale variation of standing waves patterns and the opening of an energy gap displaying an oscillating dependence on the tube length. Rather than concentrating on one particular model of a boundary, we aim to describe all possible energy independent hard wall boundary conditions for metallic single wall nanotubes. We will classify the boundary conditions in terms of their symmetry properties and show how different boundary conditions produce varying degrees of K-point degeneracy breaking.
In the scanning tunneling microscopy measurements of Ref. 6 an additional slow spatial modulation of the standing waves was observed. It was interpreted as being a beating envelope function with wavevector $\mathbf{q},|\mathbf{q}| \ll|\mathbf{K}|$, resulting from the interference of left and right moving waves with slightly different total wavevectors $\mathbf{K} \pm \mathbf{q}$. Theoretically, the effective mass model $[15,16,17,18]$ provides a reliable analytical description of the electronic structure near the $\mathrm{K}$ point where the total wavevector is $\mathbf{k}=\mathbf{K}+\mathbf{q}$ and the dispersion relation is linear $E=s v|\mathbf{q}|$, $v$ is the Fermi velocity and $s= \pm 1$ for the conduction and valence band, respectively. For spinless electrons, the envelope wavefunction $\Psi(\mathbf{q}, \mathbf{r})$ has four components corresponding to two inequivalent atomic sites in the hexagonal graphite lattice ("A" and " $\mathrm{B}$ ") and to two inequivalent K-points in the hexagonal first Brillouin zone. The resulting eigenvalue equation for $\Psi$ is the massless Dirac equation,

$$
\begin{aligned}
-i v \alpha \cdot \nabla \Psi & =E \Psi ; \quad \alpha=\left(\begin{array}{cc}
\sigma & 0 \\
0 & -\sigma
\end{array}\right) \\
\sigma & =e^{i \eta \sigma_{z} / 2}\left(\sigma_{x} \hat{\imath}+\sigma_{y} \hat{\jmath}\right) e^{-i \eta \sigma_{z} / 2}
\end{aligned}
$$

where the role of spin ("pseudo-spin") is assumed by the relative amplitudes on the $\mathrm{A}$ and $\mathrm{B}$ atomic sites: $\sigma$ is a vector in the $(x, y)$ plane rotated by the chiral angle $\eta$ of the tube. Also, $v=(\sqrt{3} / 2) a \gamma$ is the Fermi velocity, $a$ is the lattice constant of graphite and $\gamma$ is the nearest neighbour transfer integral.

In this paper we consider the effective boundary conditions for the envelope function $\Psi$ in a finite size carbon nanotube. Since the effective mass model for $\Psi$ corresponds to the Dirac equation, we begin by deriving all possible energy independent hard wall boundary conditions for the Dirac equation. We write them in terms of a small number of arbitrary parameters, mixing angles, that describe mixing between boundary conditions with different discrete symmetries. Then, in order to illustrate the meaning of the general boundary conditions, we evaluate the resulting energy level spectra for non-interacting electrons in finite-length metallic nanotubes and the corresponding standing wave envelope functions. To anticipate a little, we find that energy independent hard wall 
boundary conditions for the Dirac equation may be expressed in general terms as

$$
\Psi=M \Psi ; \quad M^{2}=1 ; \quad\left\{\mathbf{n}_{\mathbf{B}} \cdot \alpha, M\right\}=0,
$$

where $M$ is an Hermitian, unitary $4 \times 4$ matrix $M^{2}=1$ with the constraint that it anticommutes with the operator $\mathbf{n}_{\mathbf{B}} \cdot \alpha$, proportional to the component of the current operator normal to the interface, $\mathbf{n}_{\mathbf{B}}$ is the unit vector normal to the interface. As explained in the Appendix, we find four possible linear combinations of matrices satisfying these constraints on $M$, which, assuming $\mathbf{n}_{\mathbf{B}}$ is a vector confined to the $(x, y)$ plane, may be written in terms of a small number of arbitrary parameters:

$$
\begin{aligned}
& M_{1}=\cos \Lambda\left(I_{\Pi} \otimes \mathbf{n}_{1} \cdot \sigma\right)+\sin \Lambda\left(\Pi_{z} \otimes \mathbf{n}_{\mathbf{2}} \cdot \sigma\right) \\
& M_{2}=\cos \Upsilon\left(\nu_{\mathbf{1}} \cdot \boldsymbol{\Pi} \otimes I_{\sigma}\right)+\sin \Upsilon\left(\nu_{\mathbf{2}} \cdot \boldsymbol{\Pi} \otimes \mathbf{n}_{\mathbf{B}} \cdot \sigma\right) \\
& M_{3}=\cos \Omega\left(\nu_{\mathbf{2}} \cdot \boldsymbol{\Pi} \otimes \mathbf{n}_{\mathbf{B}} \cdot \sigma\right)+\sin \Omega\left(I_{\Pi} \otimes \mathbf{n}_{\mathbf{1}} \cdot \sigma\right) \\
& M_{4}=\cos \Theta\left(\nu_{\mathbf{1}} \cdot \boldsymbol{\Pi} \otimes I_{\sigma}\right)+\sin \Theta\left(\Pi_{z} \otimes \mathbf{n}_{\mathbf{2}} \cdot \sigma\right)
\end{aligned}
$$

where the angles $\Lambda, \Upsilon, \Theta$ and $\Omega$ are arbitrary, $\mathbf{n}_{\mathbf{1}}$ and $\mathbf{n}_{\mathbf{2}}$ are three-dimensional space-like vectors satisfying the contraints $\mathbf{n}_{\mathbf{1}} \cdot \mathbf{n}_{\mathbf{B}}=\mathbf{n}_{\mathbf{2}} \cdot \mathbf{n}_{\mathbf{B}}=\mathbf{n}_{\mathbf{1}} \cdot \mathbf{n}_{\mathbf{2}}=0$, and $\nu_{\mathbf{1}}$ and $\nu_{\mathbf{2}}$ are two-dimensional (confined to the $(x, y)$ plane) spacelike vectors satisfying the constraint $\nu_{\mathbf{1}} \cdot \nu_{\mathbf{2}}=0$. Here we have adopted a matrix direct product notation to highlight the separate $\mathrm{K}$-point space and $\mathrm{AB}$ space structure, using the notation $\left\{\sigma_{x}, \sigma_{y}, \sigma_{z}, I_{\sigma}\right\}$ for $2 \times 2$ Pauli matrices and the unit matrix that operate within a block ('AB space') and $\left\{\Pi_{x}, \Pi_{y}, \Pi_{z}, I_{\Pi}\right\}$ for $2 \times 2$ Pauli matrices and the unit matrix that operate in K-point space. For example, the operator $\alpha$ may be written as a direct product $\alpha=\Pi_{z} \otimes \sigma$. Note that the boundary conditions of the Hadron bag model[19], in which elementary particles are confined by a scalar (mass) term at the boundary, are described by $M=\nu_{\mathbf{2}} \cdot \boldsymbol{\Pi} \otimes \mathbf{n}_{\mathbf{B}} \cdot \sigma$. Berry and Mondragon [20] considered "neutrino billiards" with a two component Dirac equation confined by a term proportional to $\sigma_{z}$, corresponding to $M=I_{\Pi} \otimes \mathbf{n}_{1} . \sigma$ or $M=\Pi_{z} \otimes \mathbf{n}_{\mathbf{2}} . \sigma$ with either $\mathbf{n}_{\mathbf{1}}$ or $\mathbf{n}_{\mathbf{2}}$ lying in the $(x, y)$ plane.

There are two non-equivalent K-points that we label as $K$ and $\widetilde{K}$. The Dirac equation is diagonal in K-point space, so that, in the absence of boundary conditions, there are two right moving $\left(\Psi_{K}^{(R)}\right.$ and $\left.\Psi_{\widetilde{K}}^{(R)}\right)$ and two left moving $\left(\Psi_{K}^{(L)}\right.$ and $\left.\Psi_{\widetilde{K}}^{(L)}\right)$ plane wave solutions near the Fermi surface of a metallic tube. The solutions $\Psi_{K}^{(R)}$ and $\Psi_{\widetilde{K}}^{(L)}$ are eigenvectors of the pseudo-spin component along the tube axis $\boldsymbol{\Sigma} . \mathbf{n}_{\mathbf{B}}$ with eigenvalue $+s$, whereas the solutions $\Psi_{\widetilde{K}}^{(R)}$ and $\Psi_{K}^{(L)}$ have eigenvalue $-s$, where $s= \pm 1$ for the conduction and valence band, respectively. Also, the solutions $\Psi_{K}^{(R)}$ and $\Psi_{K}^{(L)}$ are eigenvectors of pseudohelicity $-i \boldsymbol{\Sigma} . \nabla /|\mathbf{q}|$ with eigenvalue $+s$, whereas the solutions $\Psi_{\widetilde{K}}^{(R)}$ and $\Psi_{\widetilde{K}}^{(L)}$ have eigenvalue $-s$. There are different ways of combining the right and left moving waves in order to create standing waves. The first possibility is that waves at the same K-point combine, namely $\Psi_{K}^{(R)}$ and $\Psi_{K}^{(L)}$ form a standing wave with helicity eigenvalue $+s$, and $\Psi_{\widetilde{K}}^{(R)}$ and $\Psi_{\widetilde{K}}^{(L)}$ form a standing wave with helicity eigenvalue $-s$. This situation is realised by the matrix $M_{1}$, Eq.(3), because it is diagonal in K-point space. A second possibility is that waves from opposite K-points combine, namely $\Psi_{K}^{(R)}$ and $\Psi_{\widetilde{K}}^{(L)}$ form a standing wave with spin component eigenvalue $+s$, and $\Psi_{\widetilde{K}}^{(R)}$ and $\Psi_{K}^{(L)}$ form a standing wave with spin component eigenvalue $-s$. This situation is realised by the matrix $M_{2}$, Eq.(4), because it is off-diagonal in K-point space. A third possibility is a combination of the previous two, with waves scattered back at the boundary into a mixture of both of the K-points. This situation is realised by the matrices $M_{3}$, Eq.(5), and $M_{4}$, Eq.(6), because they have both diagonal and off-diagonal in K-point space parts.

\section{EFFECTIVE MASS MODEL}

In the effective mass model of two-dimensional graphite [15], the total wavefunction $\Psi_{\text {tot }}$ is written as a linear combination of four components $m=\{1,2,3,4\}$ corresponding to two K-points $\mu=\{1,2\}$ and $\pi$-type atomic orbitals $\varphi_{j}\left(\mathbf{r}-\mathbf{R}_{\mathbf{j}}\right)$ on two non-equivalent atomic sites $j=\{A, B\}$ in the unit cell,

$$
\Psi_{t o t}(\mathbf{r})=\sum_{m=1}^{4}\left\{\Phi_{m}^{(0)}(\mathbf{r})-\mathbf{G}_{m}(\mathbf{r}) . \nabla+\ldots\right\} \psi_{m}(\mathbf{r})
$$

where

$$
\begin{aligned}
\Phi_{m}^{(0)}(\mathbf{r}) & =\frac{1}{\sqrt{N}} \sum_{\mathbf{R}_{\mathbf{j}}}^{N} e^{i \mathbf{K}_{\mu} \cdot \mathbf{R}_{\mathbf{j}}} \varphi_{j}\left(\mathbf{r}-\mathbf{R}_{j}\right) \\
\mathbf{G}_{m}(\mathbf{r}) & =\frac{1}{\sqrt{N}} \sum_{\mathbf{R}_{\mathbf{j}}}^{N} e^{i \mathbf{K}_{\mu} \cdot \mathbf{R}_{\mathbf{j}}} \varphi_{j}\left(\mathbf{r}-\mathbf{R}_{j}\right)\left(\mathbf{r}-\mathbf{R}_{j}\right),
\end{aligned}
$$

are Bloch type functions constructed from the atomic orbitals, $\mathbf{R}_{j}$ is the position of an atom in real space and the summation is over the number of unit cells $N \gg 1$. The functions $\psi_{m}(\mathbf{r})$ are components of the envelope function $\Psi(\mathbf{q}, \mathbf{r})$. Substituting this expression for $\Psi_{\text {tot }}$ into the Schrödinger equation and integrating with respect to fast degrees of freedom that vary on the scale of the unit cell leads to the Dirac equation Eq.(2) for the envelope function $\Psi$ where the K-points are taken as $\mathbf{K}=( \pm 4 \pi / 3 a, 0)$ and the components of $\Psi$ are written in the order $K A$, $K B, \widetilde{K} B, \widetilde{K} A$. The appearance of the chiral angle of the tube $\eta$ in the Dirac equation shows that the axes of the $(x, y)$ coordinate system have been rotated to be transverse and parallel to the tube axis. Applying periodic boundary conditions to the wavefunction $\Psi_{t o t}$, Eq. (7), in the direction transverse to the nanotube axis produces a 
condition for the envelope function $\Psi$ that leads to metallic or semiconducting behaviour depending on whether the transverse component of wavevector $\mathbf{q}$ is allowed to be zero $[16,17]$.

\section{BOUNDARY CONDITIONS FOR THE EFFECTIVE MASS MODEL}

In order to obtain hard wall boundary conditions for the Dirac equation, we place an additional confinement potential at the boundary $\mathbf{r}=\mathbf{r}_{B}$,

$$
\left[-i v \alpha \cdot \nabla+c v \widetilde{M} \delta\left(\mathbf{r}-\mathbf{r}_{\mathbf{B}}\right)\right] \Psi=E \Psi
$$

where $c$ is a real constant and $\widetilde{M}$ is an arbitrary $4 \times 4$ Hermitian, unitary matrix, $\widetilde{M}^{2}=1$. The orientation of the boundary is defined by a unit vector $\mathbf{n}_{B}$ normal to it, and we assume that the wavefunction is zero outside the confined region, but non-zero inside it. Then, we integrate across an infinitesimal width of the boundary, giving

$$
-i \mathbf{n}_{\mathbf{B}} \cdot \alpha \Psi=c \widetilde{M} \Psi
$$

Substituting this equation back into itself, we find the requirements that $c^{2} \widetilde{M}^{2}=1$ (thus we set $c=1$ ) and $\left\{\mathbf{n}_{\mathbf{B}} \cdot \alpha, \widetilde{M}\right\}=0$. The boundary condition can be written as $\Psi=M \Psi$ where $M=i \mathbf{n}_{\mathrm{B}} \cdot \alpha \widetilde{M}, M^{2}=1$ and $\left\{\mathbf{n}_{\mathbf{B}} \cdot \alpha, M\right\}=0$, giving the result quoted in the introduction Eq. (2).

If, in the graphite coordinate system, we define the normal to the boundary as $\mathbf{n}_{\mathbf{B}}=(\sin \eta, \cos \eta, 0)$, where $\eta$ is the chiral angle of the tube, then we may choose two mutually orthogonal $3 \mathrm{D}$ vectors as $\mathbf{n}_{\mathbf{1}}=(\cos \eta \sin \zeta,-\sin \eta \sin \zeta, \cos \zeta)$ and $\mathbf{n}_{\mathbf{2}}=$ ( $\cos \eta \cos \zeta,-\sin \eta \cos \zeta,-\sin \zeta$ ), and two additional orthogonal $2 \mathrm{D}$ vectors as $\nu_{\mathbf{1}}=(\cos \xi, \sin \xi, 0)$ and $\nu_{\mathbf{2}}=$ $(-\sin \xi, \cos \xi, 0)$. This introduces two new mixing angles, $\zeta$ and $\xi$ : the arbitrary parameters contained within the boundary conditions describe the amount of mixing between different discrete symmetries. First, we note that the pseudo-spin of a $2 \mathrm{D}$ graphite sheet does not transform in the same way as the spin of relativistic fermions, because certain transformations result in a swapping of the orientation of $\mathrm{A}$ and $\mathrm{B}$ atoms. This additional operation is described by a "pseudo-spin-flip" operator $\rho_{z}=\Pi_{x} \otimes i \sigma_{z}$ that corresponds to a reflection in the $(x, y)$ plane of relativistic fermions. For example, an active rotation of the $2 \mathrm{D}$ graphite sheet anticlockwise by $\pi / 3$ about the perpendicular $z$ axis, $\Psi\left(\mathbf{r}^{\prime}\right)=C_{6} \Psi(\mathbf{r})$, is described by $C_{6}=\rho_{z} R(\pi / 3)=\Pi_{x} \otimes \exp \left((2 \pi i / 3) \sigma_{z}\right)$ where $R(\theta)=I_{\Pi} \otimes \exp \left((i \theta / 2) \sigma_{z}\right)$ is a continuous rotation operator.

Table 1 shows a summary of the discrete symmetries of the boundary conditions in terms of the orientation of the vectors $\mathbf{n}_{\mathbf{1}}, \mathbf{n}_{\mathbf{2}}, \nu_{\mathbf{1}}$ and $\nu_{\mathbf{2}}$. In addition to $\rho_{z}$ we consider parity $P=\Pi_{x} \otimes I_{\sigma}$, corresponding to a rotation by $\pi$ about the $z$ axis $(x \rightarrow-x$ and $y \rightarrow-y)$, and charge conjugation $(C)$ and time reversal symmetry $(T)$ that involve the complex conjugation operator combined with $C=-i \Pi_{y} \otimes \sigma_{y}$ and $T=I_{\Pi} \otimes \sigma_{y}$, respectively. The angles $\zeta$ and $\xi$ mix terms with different symmetry with respect to $\rho_{z}: \zeta=0$ and $\xi=0$ correspond to evenness with respect to $\rho_{z}$ whereas $\zeta=\pi / 2$ and $\xi=\pi / 2$ correspond to oddness. Since spin and/or helicity label different states at the same energy, values of $\zeta$ and $\xi$ not equal to multiples of $\pi / 2$ will lead to broken degeneracy. The angles $\Lambda, \Upsilon, \Theta$ and $\Omega$ mix different symmetries with respect to combinations of $P, C$ and $\rho_{z}$.

\begin{tabular}{|c|c|c|c|c|c|c|}
\hline \hline$M$ & & & $\rho_{z}$ & $P$ & $C$ & $T$ \\
\hline \hline$I_{\Pi} \otimes \mathbf{n}_{\mathbf{1}} \cdot \sigma$ & $\mathbf{n}_{\mathbf{1}}=\mathbf{n}_{(x, y)}$ & $\zeta_{u}=\frac{\pi}{2}$ & -1 & +1 & +1 & -1 \\
\hline & $\mathbf{n}_{\mathbf{1}}=\mathbf{n}_{z}$ & $\zeta_{u}=0$ & +1 & +1 & +1 & -1 \\
\hline$\Pi_{z} \otimes \mathbf{n}_{\mathbf{2}} \cdot \sigma$ & $\mathbf{n}_{\mathbf{2}}=\mathbf{n}_{(x, y)}$ & $\zeta_{u}=0$ & +1 & -1 & -1 & -1 \\
\hline & $\mathbf{n}_{\mathbf{2}}=\mathbf{n}_{z}$ & $\zeta_{u}=\frac{\pi}{2}$ & -1 & -1 & -1 & -1 \\
\hline$\nu_{\mathbf{1}} \cdot \boldsymbol{\Pi} \otimes I_{\sigma}$ & $\nu_{\mathbf{1}}=\hat{\imath}$ & $\xi_{u}=0$ & +1 & +1 & +1 & +1 \\
\hline & $\nu_{\mathbf{1}}=\hat{\jmath}$ & $\xi_{u}=\frac{\pi}{2}$ & -1 & -1 & +1 & -1 \\
\hline$\nu_{\mathbf{2}} \cdot \boldsymbol{\Pi} \otimes \mathbf{n}_{\mathbf{B}} \cdot \sigma$ & $\nu_{\mathbf{2}}=\hat{\imath}$ & $\xi_{u}=-\frac{\pi}{2}$ & -1 & +1 & -1 & -1 \\
\hline & $\nu_{\mathbf{2}}=\hat{\jmath}$ & $\xi_{u}=0$ & +1 & -1 & -1 & +1 \\
\hline \hline
\end{tabular}

Discrete symmetries of the boundary conditions

\section{SINGLE PARTICLE ENERGY SPECTRUM}

In order to illustrate the meaning of the general boundary conditions, we calculate the form of non-interacting single particle standing waves created by the boundary conditions and the corresponding energy spectrum. For simplicity, we will consider only metallic nanotubes with arbitrary chiral angle $\eta$. We suppose that the $x$ axis is perpendicular to the tube axis and we consider only the zero momentum transverse mode so that $|E|<2 \pi v /\left|\mathbf{C}_{\mathbf{h}}\right|$ where $\left|\mathbf{C}_{\mathbf{h}}\right|$ is the circumference. The Dirac equation is diagonal in K-point space, so that, in the absence of boundary conditions, there are two right moving $\left(\Psi_{K}^{(R)}\right.$ and $\left.\Psi_{\widetilde{K}}^{(R)}\right)$ and two left moving $\left(\Psi_{K}^{(L)}\right.$ and $\left.\Psi_{\widetilde{K}}^{(L)}\right)$ plane wave solutions:

$\Psi_{K}^{(R)}=A e^{i q y}\left(\begin{array}{c}1 \\ i s e^{-i \eta} \\ 0 \\ 0\end{array}\right) ; \Psi_{K}^{(L)}=B e^{-i q y}\left(\begin{array}{c}1 \\ -i s e^{-i \eta} \\ 0 \\ 0\end{array}\right) ;$
$\Psi_{\widetilde{K}}^{(R)}=C e^{i q y}\left(\begin{array}{c}0 \\ 0 \\ 1 \\ -i s e^{-i \eta}\end{array}\right) ; \Psi_{\widetilde{K}}^{(L)}=D e^{-i q y}\left(\begin{array}{c}0 \\ 0 \\ 1 \\ i s e^{-i \eta}\end{array}\right)$, 
where $A, B, C$ and $D$ are arbitrary constants, $q$ is the wavevector along the tube and we consider $q \geq 0$ and $E=s v q, s= \pm 1$. The solutions $\Psi_{K}^{(R)}$ and $\Psi_{\widetilde{K}}^{(L)}$ are eigenvectors of pseudo-spin component $\boldsymbol{\Sigma} \cdot \hat{\jmath}=$ $I_{\Pi} \otimes e^{i \eta \sigma_{z} / 2} \sigma_{y} e^{-i \eta \sigma_{z} / 2}$ with eigenvalue $+s$, whereas the solutions $\Psi_{\widetilde{K}}^{(R)}$ and $\Psi_{K}^{(L)}$ have eigenvalue $-s$. Also, the solutions $\Psi_{K}^{(R)}$ and $\Psi_{K}^{(L)}$ are eigenvectors of pseudohelicity $-i \boldsymbol{\Sigma} . \nabla /|\mathbf{q}|=|\mathbf{q}|^{-1} I_{\Pi} \otimes e^{i \eta \sigma_{z} / 2}\left(-i \sigma_{y} \partial_{y}\right) e^{-i \eta \sigma_{z} / 2}$ with eigenvalue $+s$, whereas the solutions $\Psi_{\widetilde{K}}^{(R)}$ and $\Psi_{\widetilde{K}}^{(L)}$ have eigenvalue $-s$. In the following we consider each of the four linear combinations $M_{1}$ to $M_{4}$ separately, and we consider a system with the same type of boundary condition on the right (at $y=+L / 2$ ) and on the left (at $y=-L / 2$ ). We introduce an index $u=\{R, L\} \equiv \pm 1$ to label the right and left hand side so that the normal to the boundary, defined with respect to the graphite coordinate system, is $\mathbf{n}_{\mathbf{B}}=u(\sin \eta, \cos \eta, 0)$, and we take into account the possibility of different mixing angles, $\Lambda_{u}, \Upsilon_{u}, \Theta_{u}$ and $\Omega_{u}$, and vectors $\mathbf{n}_{\mathbf{1}}=\left(u \cos \eta \sin \zeta_{u},-u \sin \eta \sin \zeta_{u}, \cos \zeta_{u}\right)$, $\mathbf{n}_{\mathbf{2}}=\left(u \cos \eta \cos \zeta_{u},-u \sin \eta \cos \zeta_{u},-\sin \zeta_{u}\right), \quad \nu_{\mathbf{1}}=$ $\left(\cos \xi_{u}, \sin \xi_{u}, 0\right)$ and $\nu_{2}=\left(-\sin \xi_{u}, \cos \xi_{u}, 0\right)$.

\section{M1: diagonal boundary conditions}

With the above definitions of the mixing angles, the boundary condition $\Psi=M_{1} \Psi$ produces the following relations between the components of the wavefunction at the interface:

$u \sin \left(\zeta_{u}+\Lambda_{u}\right) e^{-i \eta} \psi_{A K}-\left[1+\cos \left(\zeta_{u}+\Lambda_{u}\right)\right] \psi_{B K}=0$, $u \sin \left(\zeta_{u}-\Lambda_{u}\right) e^{+i \eta} \psi_{A \widetilde{K}}-\left[1-\cos \left(\zeta_{u}-\Lambda_{u}\right)\right] \psi_{B \widetilde{K}}=0$.

The equations are diagonal in K-point space so do not describe intervalley scattering. With these boundary conditions on the right (at $y=+L / 2$ ) and on the left (at $y=-L / 2$ ), standing waves at $K$ are created from combining $\Psi_{K}^{(R)}$ and $\Psi_{K}^{(L)}$ and are labelled by helicity $\lambda=+s$, and those at $\widetilde{K}$ are created from $\Psi_{\widetilde{K}}^{(R)}$ and $\Psi_{\widetilde{K}}^{(L)}$ and have label $\lambda=-s$. We find that

$B=(-1)^{p_{1}} A \exp \left[i s\left(\zeta_{R}-\zeta_{L}\right) / 2+i s\left(\Lambda_{R}-\Lambda_{L}\right) / 2\right]$,

$D=(-1)^{p_{2}} C \exp \left[-i s\left(\zeta_{R}-\zeta_{L}\right) / 2+i s\left(\Lambda_{R}-\Lambda_{L}\right) / 2\right]$,

and the corresponding wavevectors are

$$
\begin{aligned}
& q^{(\lambda=+s)}=-\frac{s\left(\zeta_{R}+\zeta_{L}\right)}{2 L}-\frac{s\left(\Lambda_{R}+\Lambda_{L}\right)}{2 L}+\frac{\pi p_{1}}{L} \\
& q^{(\lambda=-s)}=+\frac{s\left(\zeta_{R}+\zeta_{L}\right)}{2 L}-\frac{s\left(\Lambda_{R}+\Lambda_{L}\right)}{2 L}+\frac{\pi p_{2}}{L}
\end{aligned}
$$

where $\left\{p_{1}, p_{2}\right\}$ are integers such that $q \geq 0$. Using $E=$ $s v q$ shows that the mixing angles $\zeta_{R}$ and $\zeta_{L}$ break Kpoint degeneracy whereas $\Lambda_{R}$ and $\Lambda_{L}$ break electron-hole symmetry.

\section{M2: off-diagonal boundary conditions}

The boundary condition $\Psi=M_{2} \Psi$ is equivalent to the following relations between the components of the enveloped wavefunction at the interface:

$$
\begin{aligned}
& \psi_{A K}+u \sin \Upsilon_{u} e^{+i \eta-i \xi_{u}} \psi_{A \widetilde{K}}-\cos \Upsilon_{u} e^{-i \xi_{u}} \psi_{B \widetilde{K}}=0 \\
& \psi_{B K}-u \sin \Upsilon_{u} e^{-i \eta-i \xi_{u}} \psi_{B \widetilde{K}}-\cos \Upsilon_{u} e^{-i \xi_{u}} \psi_{A \widetilde{K}}=0
\end{aligned}
$$

The equations are off-diagonal in $\mathrm{K}$ space so describe intervalley scattering. Standing waves are created from combining $\Psi_{K}^{(R)}$ and $\Psi_{\widetilde{K}}^{(L)}$, with spin eigenvalue $\Sigma=+s$, and $\Psi_{\widetilde{K}}^{(R)}$ and $\Psi_{K}^{(L)}$, with spin eigenvalue $\Sigma=-s$. We find that

$$
\begin{aligned}
D & =(-1)^{p_{1}} A \exp \left[i s\left(\Upsilon_{R}-\Upsilon_{L}\right) / 2+i s\left(\xi_{R}+\xi_{L}\right) / 2\right] \\
B & =(-1)^{p_{2}} C \exp \left[i s\left(\Upsilon_{R}-\Upsilon_{L}\right) / 2-i s\left(\xi_{R}+\xi_{L}\right) / 2\right]
\end{aligned}
$$

and the corresponding wavevectors are

$$
\begin{aligned}
& q^{(\Sigma=+s)}=-\frac{s\left(\Upsilon_{R}+\Upsilon_{L}\right)}{2 L}-\frac{\left(\xi_{R}-\xi_{L}\right)}{2 L}+\frac{\pi p_{1}}{L} \\
& q^{(\Sigma=-s)}=-\frac{s\left(\Upsilon_{R}+\Upsilon_{L}\right)}{2 L}+\frac{\left(\xi_{R}-\xi_{L}\right)}{2 L}+\frac{\pi p_{2}}{L}
\end{aligned}
$$

where $\left\{p_{1}, p_{2}\right\}$ are integers such that $q \geq 0$. The angles $\xi_{R}$ and $\xi_{L}$ break degeneracy whereas $\Upsilon_{R}$ and $\Upsilon_{L}$ break electron-hole symmetry.

\section{M3: mixed boundary conditions (i)}

The boundary condition $\Psi=M_{3} \Psi$ produces the following relations between the components of the wavefunction at the interface:

$$
\begin{aligned}
& \psi_{A K}\left(1-\sin \Omega_{u} \cos \zeta_{u}\right)+u \cos \Omega_{u} e^{+i \eta-i \xi_{u}} \psi_{A \widetilde{K}} \\
& \quad-u \sin \Omega_{u} \sin \zeta_{u} e^{+i \eta} \psi_{B K}=0, \\
& \psi_{B K}\left(1+\sin \Omega_{u} \cos \zeta_{u}\right)-u \cos \Omega_{u} e^{-i \eta-i \xi_{u}} \psi_{B \widetilde{K}} \\
& \quad-u \sin \Omega_{u} \sin \zeta_{u} e^{-i \eta} \psi_{A K}=0 .
\end{aligned}
$$

The matrix $M_{3}$ has both diagonal and off-diagonal in Kpoint space parts. Standing waves are created from linear combinations of all $\Psi_{K}^{(R)}, \Psi_{\widetilde{K}}^{(L)}, \Psi_{\widetilde{K}}^{(R)}$ and $\Psi_{K}^{(L)}$. We find that

$$
\begin{aligned}
& B=\sin \Omega_{u} e^{i s u \zeta_{u}} A e^{i u q L}+i s u \cos \Omega_{u} e^{-i \xi_{u}} C e^{i u q L}, \\
& D=\sin \Omega_{u} e^{-i s u \zeta_{u}} C e^{i u q L}+i s u \cos \Omega_{u} e^{+i \xi_{u}} A e^{i u q L} .
\end{aligned}
$$

and the corresponding wavevectors are given by

$$
\begin{aligned}
& \cos (2 q L)=\cos \beta ; \\
& \cos \beta=\sin \Omega_{R} \sin \Omega_{L} \cos \left(\zeta_{R}+\zeta_{L}\right) \\
& \quad-\cos \Omega_{R} \cos \Omega_{L} \cos \left(\xi_{R}-\xi_{L}\right),
\end{aligned}
$$


where $q \geq 0$ and $0 \leq \beta \leq \pi$. The energy levels are

$$
E=s v\left\{\frac{\beta}{2 L}, \frac{\pi n}{L} \pm \frac{\beta}{2 L}\right\}
$$

where $n=\{1,2,3, \ldots\}$ are integers. The spectrum always has positive-negative energy symmetry, but broken degeneracy for $\beta \neq\{0, \pi\}$.

\section{M4: mixed boundary conditions (ii)}

The boundary condition $\Psi=M_{4} \Psi$ produces the following relations between the components of the wavefunction at the interface:

$$
\begin{aligned}
& \psi_{A K}\left(1+\sin \Theta_{u} \sin \zeta_{u}\right)-u \sin \Theta_{u} \cos \zeta_{u} e^{+i \eta} \psi_{B K} \\
& \quad-\cos \Theta_{u} e^{-i \xi_{u}} \psi_{B \widetilde{K}}=0, \\
& \psi_{B K}\left(1-\sin \Theta_{u} \sin \zeta_{u}\right)-u \sin \Theta_{u} \cos \zeta_{u} e^{-i \eta} \psi_{A K} \\
& \quad-\cos \Theta_{u} e^{-i \xi_{u}} \psi_{A \widetilde{K}}=0 .
\end{aligned}
$$

The matrix $M_{4}$ has both diagonal and off-diagonal in $\mathrm{K}$ space parts. Standing waves are created from linear combinations of all $\Psi_{K}^{(R)}, \Psi_{\widetilde{K}}^{(L)}, \Psi_{\widetilde{K}}^{(R)}$ and $\Psi_{K}^{(L)}$. We find that

$$
\begin{aligned}
& B=i s u \sin \Theta_{u} e^{i s u \zeta_{u}} A e^{i u q L}+\cos \Theta_{u} e^{-i \xi_{u}} C e^{i u q L} \\
& D=i s u \sin \Theta_{u} e^{-i s u \zeta_{u}} C e^{i u q L}+\cos \Theta_{u} e^{+i \xi_{u}} A e^{i u q L}
\end{aligned}
$$

and the corresponding wavevectors are given by

$$
\begin{aligned}
& \cos (2 q L)=\cos \kappa ; \\
& \cos \kappa=\cos \Theta_{R} \cos \Theta_{L} \cos \left(\xi_{R}-\xi_{L}\right) \\
& \quad-\sin \Theta_{R} \sin \Theta_{L} \cos \left(\zeta_{R}+\zeta_{L}\right),
\end{aligned}
$$

where $q \geq 0$ and $0 \leq \kappa \leq \pi$. The energy levels are

$$
E=s v\left\{\frac{\kappa}{2 L}, \frac{\pi n}{L} \pm \frac{\kappa}{2 L}\right\}
$$

where $n=\{1,2,3, \ldots\}$ are integers. The spectrum always has positive-negative energy symmetry, but broken degeneracy for $\kappa \neq\{0, \pi\}$.

\section{DISCUSSION}

In this paper, we considered the effective mass model of spinless electrons in single wall carbon nanotubes that describes slowly varying spatial envelope wavefunctions $\Psi(\mathbf{q}, \mathbf{r})$ with small wavevectors $\mathbf{q}$ in the region of linear dispersion $E= \pm v|\mathbf{q}|$ near the K-points. Taking into account the two inequivalent $\mathrm{K}$-points, the envelope wavefunctions $\Psi$ obey the Dirac equation for massless fermions, written in terms of four component spinors, with the role of spin assumed by the relative amplitude of the wave function on the sublattice atoms ("A" and "B"). We found that energy independent hard wall boundary conditions for the Dirac equation may be written as $\Psi=M \Psi$ where $M$ is an Hermitian, unitary $4 \times 4$ matrix $M^{2}=1$ with the additional constraint that it anticommutes with the component of the current operator normal to the boundary. All possible linear combinations of matrices $M$ obeying these constraints were expressed in terms of a small number of arbitrary parameters, mixing angles, that describe mixing between boundary conditions with different discrete symmetries. Then, in order to illustrate how the presence of non-zero mixing angles breaks K-point degeneracy and electron-hole symmetry, we evaluated the resulting energy level spectra for noninteracting electrons in finite-length metallic nanotubes and the corresponding standing wave envelope functions.

The intention of this paper was to classify all possible boundary conditions for the spatially long-range envelope functions of a closed nanotube with length much greater than its circumference $L \gg L_{c}$. The analysis was restricted to energy independent boundary conditions, although in principle they could be generalised by performing a gradient expansion. We focused on armchair tubes and did not consider the possibility of edge states that may exist in zigzag graphite edges [21, 22, 23]. Rather than modelling the microscopic details of a boundary, such as shape or roughness, the nature of the boundary is characterised by mixing angles that describe the degree of symmetry breaking. In practice, the correct choice of a particular set of boundary conditions and values of symmetry mixing angles needed to describe a given nanotube will depend on experimental details and may not be known beforehand. To illustrate this, we compare our results to the idealised microscopic models of a a capped armchair nanotube and a model of a boundary obtained by setting the wavefunction to zero along a straight line of atoms. We find that the description of a capped nanotube considered in Ref. [13] corresponds to our off-diagonal boundary conditions $\mathrm{M} 2, \Psi=M_{2} \Psi$, with $\Upsilon_{u}=0$ or $\pi$ so that the component $\psi_{A K}$ of the wavefunction is related to $\psi_{B \widetilde{K}}$ at the boundary. A model of a boundary obtained by setting the wavefunction to zero, which is equivalent to a particle-in-a-box model [14], also corresponds to our off-diagonal boundary conditions M2, $\Psi=M_{2} \Psi$, but with $\Upsilon_{u}= \pm \pi / 2$ so that the component $\psi_{A K}$ of the wavefunction is related to $\psi_{A \widetilde{K}}$ at the boundary. Although the two models are described by a different mixing angle $\Upsilon$, they both correspond to the off-diagonal boundary conditions, introduce inter-valley scattering at the boundary and, in general, they break K-point degeneracy with the mixing angle $\xi_{u}$ dependent on the length of the nanotube.

The authors thank J T Chalker and C J Lambert for discussions, and EPSRC for financial support. 


\section{APPENDIX}

In this appendix, we briefly describe the method of finding linear combinations of matrices that satisfy the constraints on $M$ described by the boundary conditions, Eq. (2). Any $4 \times 4$ matrix may be written as a linear combination of the matrices $I_{\Pi} \otimes I_{\sigma}, I_{\Pi} \otimes(\mathbf{n} . \sigma),(\nu . \Pi) \otimes$ $I_{\sigma}$, and $(\nu . \boldsymbol{\Pi}) \otimes(\mathbf{n} . \sigma)$, where $\mathbf{n}$ and $\nu$ are arbitrary threedimensional space-like vectors. The first step is to find all the linear combinations that produce the unit matrix $I_{\Pi} \otimes I_{\sigma}$ when squared:

$$
\begin{aligned}
& M_{a}=I_{\Pi} \otimes I_{\sigma}, \\
& M_{b}=\cos \theta\left(I_{\Pi} \otimes \mathbf{n}_{\mathbf{1}} \cdot \sigma\right)+\sin \theta\left(\nu_{\mathbf{1}} \cdot \boldsymbol{\Pi} \otimes \mathbf{n}_{\mathbf{2}} \cdot \sigma\right), \\
& M_{c}=\cos \phi\left(\nu_{\mathbf{1}} \cdot \boldsymbol{\Pi} \otimes I_{\sigma}\right)+\sin \phi\left(\nu_{\mathbf{2}} \cdot \boldsymbol{\Pi} \otimes \mathbf{n}_{\mathbf{2}} \cdot \sigma\right),
\end{aligned}
$$

where the vectors are unit vectors with additional constraints $\mathbf{n}_{\mathbf{1}} \cdot \mathbf{n}_{\mathbf{2}}=0, \nu_{\mathbf{1}} \cdot \nu_{\mathbf{2}}=0$ that ensure no cross-terms survive. The next step is to find the conditions under which the matrices $M_{a}, M_{b}$, and $M_{c}$ anticommute with the operator $\mathbf{n}_{\mathbf{B}} \cdot \alpha$ that is proportional to the component of the current operator normal to the interface. Clearly, $M_{a}$ does not anticommute, so it is discarded. The matrix $M_{b}$ anticommutes if $\mathbf{n}_{\mathbf{1}} \cdot \mathbf{n}_{\mathbf{B}}=0, \mathbf{n}_{\mathbf{2}} \cdot \mathbf{n}_{\mathbf{B}}=0$, and $\nu_{1}=\hat{\mathbf{k}}$ (M1), or if $\mathbf{n}_{\mathbf{1}} \cdot \mathbf{n}_{\mathbf{B}}=0, \mathbf{n}_{\mathbf{2}}=\mathbf{n}_{\mathbf{B}}$, and $\nu_{1}$ is confined to the $(x, y)$ plane (M3). The matrix $M_{c}$ anticommutes if $\nu_{1}$ and $\nu_{2}$ are confined to the $(x, y)$ plane and $\mathbf{n}_{\mathbf{2}}=\mathbf{n}_{\mathbf{B}}$ (M2), or if $\nu_{1}$ is confined to the $(x, y)$ plane, $\nu_{2}=\hat{\mathbf{k}}$, and $\mathbf{n}_{\mathbf{2}} \cdot \mathbf{n}_{\mathbf{B}}=0(\mathrm{M} 4)$.

[1] Saito R, Dresselhaus G and Dresselhaus M S 1988 Physical properties of carbon nanotubes (Imperial College Press: London).

[2] Dekker C 1999 Physics Today 52 (5) 22.
[3] Bockrath M, Cobden D H, McEuen P L, Chopra N G, Zettl A, Thess A and Smalley R E 1997 Science 275 1922.

[4] Tans S J, Devoret M H, Dai H, Thess A, Smalley R E, Geerlings L J and Dekker C 1997 Nature 386474.

[5] Venema L C, Wildoer J W G, Janssen J W, Tans S J, Temminck Tuinstra H L J, Kouwenhoven L P and Dekker C 1999 Science 28352.

[6] Lemay S G, Janssen J W, van den Hout M, Mooij M, Bronikowski M J, Willis P A, Smalley R E, Kouwenhoven L P and Dekker C 2001 Nature 412617.

[7] Liang W, Bockrath M and Park H 2002 Phys. Rev. Lett. 88126801.

[8] Buitelaar M, Bachtold A, Nussbaumer T, Iqbal M and Schönenberger C 2002 Phys. Rev. Lett. 88156801.

[9] Cobden D H and Nygård J 2002 Phys. Rev. Lett. 89 046803.

[10] Rubio A, Sanchez-Portal D, Artacho E, Ordejon P and Soler J M 1999 Phys. Rev. Lett. 823520.

[11] Rochefort A, Salahub D R and Avouris P 1999 J. Phys. Chem. B 103641.

[12] Wu J, Duan W, Gu B-L, Yu J-Z and Kawazoe Y 2000 Appl. Phys. Lett. 772554.

[13] Yaguchi T and Ando T 2001 J. Phys. Soc. Japan 70 1327; Yaguchi T and Ando T 2001 J. Phys. Soc. Japan 703641.

[14] Jiang J, Dong J and Xing D Y 2002 Phys. Rev. B 65 245418.

[15] DiVincenzo D P and Mele E J 1984 Phys. Rev. B 29 1685.

[16] Ajiki H and Ando T 1993 J. Phys. Soc. Japan 621255.

[17] Kane C L and Mele E J 1997 Phys. Rev. Lett. 781932.

[18] McEuen P L, Bockrath M, Cobden D H, Yoon Y-G and Louie S G 1999 Phys. Rev. Lett. 835098.

[19] Chodos A, Jaffe R L, Johnson K, Thorn C B and Weisskopf V F 1974 Phys. Rev. D 93471.

[20] Berry M V and Mondragon R J 1987 Proc. R. Soc. Lond. A 41253.

[21] Klein, D J 1994 Chem. Phys. Lett. 217261.

[22] Fujita M, Wakabayashi K, Nakada K and Kusakabe K 1996 J. Phys. Soc. Japan 651920.

[23] Ryu S and Hatsugai Y 2002 Phys. Rev. Lett. 89077002. 